\title{
Delirium symptoms duration and mortality in SARS-COV2 elderly: results of a multicenter retrospective cohort study
}

\author{
Alessandro Morandi ${ }^{1,2}$ (1) Paola Rebora ${ }^{3}$. Gianluca Isaia ${ }^{4}$. Eleonora Grossi ${ }^{1} \cdot$ Bianca Faraci $^{1} \cdot$ Simona Gentile ${ }^{1}$. \\ Mario $\mathrm{Bo}^{4,5} \cdot$ Maria Grazia Valsecchi $^{3} \cdot$ Valentina Deiana ${ }^{6} \cdot$ Nives Ghezzi $^{6} \cdot$ Julia Miksza $^{6} \cdot$ Paolo Blangiardo $^{6}$. \\ Giuseppe Bellelli6,7
}

Received: 3 April 2021 / Accepted: 29 May 2021 / Published online: 26 June 2021

(c) The Author(s), under exclusive licence to Springer Nature Switzerland AG 2021

\begin{abstract}
Background Since the occurrence of the SARS-COV2 pandemic, there has been an increasing interest in investigating the epidemiology of delirium. Delirium is frequent in SARS-COV2 patients and it is associated with increased mortality; however, no information is available on the association between delirium duration in SARS-COV2 patients and related outcomes. Aims The aim of this study is to investigate the association between the duration of delirium symptoms and in-hospital mortality in older patients with SARS-COV2 infection.

Methods Retrospective cohort study of patients 65 years of age and older with SARS-CoV 2 infection admitted to two acute geriatric wards and one rehabilitation ward. Delirium symptoms duration was assessed retrospectively with a chart-based validated method. In-hospital mortality was ascertained via medical records.

Results A total of 241 patients were included. The prevalence of delirium on admission was $16 \%$. The median number of days with delirium symptoms was 4 (IQR 2-6.5) vs. 0 (IQR 0-2) in patients with and without delirium on admission. In the multivariable Cox regression model, each day with a delirium symptom in a patient with the same length of stay was associated with a 10\% increase in in-hospital mortality (Hazard ratio 1.1, 95\% Confidence interval 1.01-1.2; $p=0.03$ ). Other variables associated with increased risk of in-hospital death were age, comorbidity, CPAP, CRP levels and total number of drugs on admission.

Conclusions The study supports the necessity to establish protocols for the monitoring and management of delirium during emergency conditions to allow an appropriate care for older patients.
\end{abstract}

Keywords COVID $\cdot$ SARS-COV2 $\cdot$ Elderly $\cdot$ Delirium $\cdot$ Delirium symptoms

Alessandro Morandi

morandi.alessandro@gmail.com

1 Department of Rehabilitation and Aged Care, Hospital Ancelle, "Fondazione Camplani" Hospital, Via Aselli 14, 26100 Cremona, Italy

2 Parc Sanitari Pere Virgili and Vall D’Hebrón Institute of Research, Barcelona, Spain

3 Bicocca Center of Bioinformatics, Biostatistics and Bioimaging, School of Medicine and Surgery, University of Milano-Bicocca, Monza, Italy

4 Section of Geriatrics, Department of Medical Sciences, A.O.U. Città della Salute e della Scienza di Torino, Turin, Italy

5 Università degli Studi di Torino, Turin, Italy

6 Acute Geriatric Unit, San Gerardo Hospital, Monza, Italy

7 School of Medicine and Surgery, University of Milano - Bicocca, Monza, Italy

\section{Introduction}

Delirium is a serious neuropsychiatric condition characterized by acute onset, inattention, fluctuation along with other cognitive deficits and it is usually caused by a medical condition [1]. The cause of delirium is often multifactorial (e.g., infections, change in medications, use of restraints) especially in older patients [2]. In late 2019, a new emerging risk factor for delirium was the infection with SARS-COV2 [3]. The main hypothesis of the pathogenesis of delirium in patients with SARS-COV2 is a central neuroinflammation along with the worsening of other geriatric syndromes, which can be worsened by the infection itself or by the acute isolation related to the health care policies to reduce the spread of the viral infection [3]. 
It is known that delirium is frequent in older patients and its prevalence is reported between 11 and $42 \%$ in acute hospitals $[2,4,5]$. Delirium is associated with worse outcomes including longer hospital stay, increased mortality, health care costs, worsening of functional and cognitive status, and increased risk of dementia $[2,5,6]$. Delirium is also associated with patients' and caregivers' distress [7]. Importantly, researches have also shown how the duration of delirium further worsens its effects. Indeed each single day of delirium has been shown to be associated with a 10-15\% increase in mortality at 6 months and at 1 year [8-10].

Since the occurrence of the SARS-COV2 pandemic, there has been an increasing interest in investigating the epidemiology of delirium. A recent review has reported how delirium affects up to $80 \%$ of the patients with SARS-COV2 in the ICU and between 29 and $67 \%$ in non-ICU patients [3]. Delirium in patients with SARS-COV2 is reported to increase the risk of mortality and worsen patients' functional outcomes [11, 12]. However, there are no information on the effect of delirium duration on patient's health status. The assessment of delirium during the SARS-COV2 emergency has been difficult in many medical wards due to the overwhelming management of acute patients. A possible approach to investigate the duration of delirium and delirium symptoms is to retrospectively analyze medical chart for the presence of delirium symptoms, as previously described [13].

The aim of this study is to investigate the association between the duration of delirium symptoms and in-hospital mortality in older patients with SARS-COV2 infection.

\section{Methods}

\section{Study population}

This was a retrospective cohort study of patients 65 years of age and older with SARS-CoV-2 infection (positive result by SARS-CoV-2 nasopharyngeal swab polymerase chain reaction test) admitted to two acute geriatric wards of tertiary hospitals (San Gerardo hospital, in Monza and Città della Salute e della Scienza, Molinette Hospital, Torino) and one rehabilitation ward of a private hospital (Fondazione Teresa Camplani, Cremona) in Italy from February 22, 2020 to May 17, 2020.

Patients were admitted to the acute ward from the emergency department or transferred from acute hospital wards in those admitted to the rehabilitation ward. Exclusion criteria were an age younger than 65 years of age and the refusal of giving verbal consent by the patient or by a proxy on admission. The study protocol was approved by the Brianza Institutional Review Board (protocol n. 2572).

\section{Assessment of delirium and delirium symptoms duration}

The presence of delirium on admission was ascertained either by the presence of a formal assessment with the 4AT [14] or via a chart-based validated method. The 4AT was originally validated in acute geriatric wards and rehabilitation wards [14]. A recent systematic review and metaanalysis reported high sensitivity $(0.88,95 \%$ CI $0.80-0.93)$ and specificity $(0.88,95 \%$ CI $0.82-0.92)$ of the 4 AT for the assessment of delirium [15].

Delirium symptoms duration was assessed retrospectively with a chart-based validated Method [13] also used in other studies for this purpose [16, 17]. Each patient's chart was retrospectively reviewed for the daily presence of ten key words (i.e., confusion, disorientation, altered mental status, delirium, agitation, inappropriate behavior, mental status change, inattention, hallucination, lethargy). The source of information of the key words were medical, nurses, physical therapists' notes. The medical records were reviewed until patient's discharge or death or up to a maximum of 45 days. The duration of delirium symptoms was then defined according to the presence of any of the key words on each day during the hospital stay.

\section{Study outcomes}

The primary end-point was in-hospital mortality assessed through medical records.

\section{Data collection and clinical assessment}

We recorded on admission the following variables: demographics, smoking status, comorbidities (i.e., cardiac disease, hypertension, diabetes mellitus, chronic obstructive pulmonary disease-COPD, dementia), malnutrition assessed clinically, and the total number of medications. The functional status was evaluated differently in the acute geriatric wards using two items of the Katz's Activities of Daily Living (ADLs) (i.e., ability in self-bathing and self-dressing) and in the rehabilitation wards with the Barthel Index. Therefore, we created a new variable to assess patient's disability according to the presence of dependency in self-bathing or self-dressing or to a Barthel Index score $\leq 90 / 100$ with reference to one month before the hospitalization for SARS-COV2 infection [11].

We recorded on admission laboratory exams (white blood cell count; C-reactive protein, CRP; albumin), the use of oxygen and continuous positive airway pressure 
(CPAP) for respiratory support, the presence and the degree of chest infiltrates on chest X-ray or CT scan.

\section{Statistical analysis}

Continuous variables were described with median and interquartile range (IQR), and categorical data were expressed as frequency and percentage. Clinical features of the patients with and without delirium on admission were compared by Mann-Whitney test and Fisher test for continuous and categorical data, respectively.

The percentage of days with delirium symptoms was computed for each patient as the total number of days with delirium symptoms divided by the total number of days in the ward. The distribution of the percentage of days was reported by a violin plot (kernel density) over-imposed on a box-plot by in-hospital discharge status (dead, alive).

To evaluate the association between the percentage of days with delirium symptoms during hospital stay and inhospital mortality we applied a time-dependent multivariable Cox regression model stratified for center, in which the variable number of days with delirium is updated every day according to delirium status by a cumulative sum. The percentage of delirium days was computed as the number of delirium days divided by the length of stay to standardize for length of stay that could indeed be influenced by many factors, including delirium. The time-dependent Cox model adjusts for this potential bias by considering the number of patients at risk each day. The time origin is the day of ward's admission and patient is followed up to death or discharge, whichever occurs first. Patients transferred in other wards were followed up to hospital discharge but on the purpose of this analysis were censored at transfer. The model included potential confounders selected by generalized linear model with lasso penalty: sex, age, number of chronic diseases, cardiac disease, COPD, use of CPAP, number of chronic drugs, chest X-ray or CT, CRP serum levels. The shrinkage parameter was set as the one minimizing the mean prediction error by a tenfold cross validation. Proportional hazard assumption was fulfilled. Results were obtained using SAS (version 9.4) and R software (version 3.5.2).

\section{Results}

A total of 241 patients were included, 203 admitted to acute geriatric wards, and 38 to a rehabilitation ward.

The prevalence of delirium on admission was $39 / 241=16 \%$. Patients with delirium on admission were older, with higher number of comorbidities and higher prevalence of dementia. Functional disability, before the index admission, was higher in patients with delirium (Table 1).
Overall, we observed a total number of days with delirium symptoms of 566 out of 3886 hospitalization days: thus, days with delirium were $15 \%$ of total days of hospitalization. The median number of days with delirium symptoms was higher in subjects with delirium at admission (4, IQR 2-6.5, vs. 0, IQR 0-2). In patients with delirium at admission, the median percentage of days of delirium symptoms per days of hospital stay was 50\% (IQR 6.3-67.3\%).

A total of 77 in-hospital deaths were observed, 20 (51\%) in patients with delirium at admission and $57(28 \%)$ in the others $(p=0.008)$. Patients who died in the hospital were older, with higher prevalence of malnutrition, smoking history, dementia, functional disability, cardiac disease and overall a higher number of comorbidities and medications (Table 2). Likewise, the degree of the infection was more severe in patients who died than in the others, as indicated by a higher prevalence of single or bilateral lung opacities and by the need of Oxygen support or CPAP use. The median number of delirium symptoms days in those who died was 2 (IQR 0.0-4.0) and the percentage of days of delirium symptoms per days of hospital stay was 22 (IQR 0.0-60). Figure 1 shows the distribution of the percentage of days with delirium symptoms according to in-hospital mortality, showing that the majority of alive patients did not show any delirium symptoms during hospital stay, while patients who died had higher percentage of days with delirium symptoms.

In the multivariable Cox regression model, each day with a delirium symptom in a patient with the same length of stay was associated with a $10 \%$ increase in in-hospital mortality (Hazard ratio 1.1, 95\% confidence interval 1.01-1.2; $p=0.03)$. Other variables associated with increased risk of in-hospital death were age (HR 1.03, 95\% CI 1-1.06; $p=0.05$ ), comorbidity (HR 1.3, 95\% CI 1.03-1.5; $p=0.025$ ), CPAP (HR 2.11, 95\% CI 1.11-4.02; $p=0.02$ ), CRP levels (HR 1.08, 95\% CI 1.04-1.12; $p<0.001$ ) and total number of drugs (HR 1.13, 95\% CI 1.04-1.23; $p=p<0.001$ ) on admission (Table 3).

\section{Discussion}

The study confirms previous data on the prevalence of delirium on admission in acutely ill older patients and provides new information on the duration of delirium symptoms. Forty nine percent of the patients experienced at least 1 day with delirium symptoms and each day with a delirium symptom in a patient with the same length of stay was associated with a $10 \%$ increase in in-hospital mortality.

Previous studies have reported delirium prevalence in non-ICU SARS-COV2 patients between 29 and 67\% [3]. In this population, it was confirmed that delirium on admission is associated with increased mortality and length of hospital stay. [3, 11, 18] A large Italian multicenter study showed that 
Table 1 Patients' characteristics by delirium at admission

\begin{tabular}{|c|c|c|c|c|c|}
\hline Variable* & Entire sample & $\begin{array}{l}\text { No delirium } \\
N=202\end{array}$ & $\begin{array}{l}\text { Delirium } \\
N=39\end{array}$ & $p$ value & Missing $\%$ \\
\hline Age & $77.5[65.6,85.0]$ & $76.8[64.5,84.1]$ & $83.3[79.1,87.6]$ & 0.001 & 0 \\
\hline Gender (male), $N(\%)$ & $143(59)$ & $125(62)$ & $18(46)$ & 0.076 & 0 \\
\hline Smoking (current or previous), $N(\%)$ & $61(35)$ & $53(34)$ & $8(38)$ & 0.808 & 27 \\
\hline Cardiac diseases, $N(\%)^{* *}$ & $93(39)$ & 75 ( 37$)$ & $18(46)$ & 0.369 & 0 \\
\hline Hypertension, $N(\%)$ & $143(59)$ & $118(58)$ & $25(64)$ & 0.595 & 0 \\
\hline Diabetes mellitus, $N(\%)$ & $57(24)$ & $43(21)$ & $14(36)$ & 0.063 & 0 \\
\hline $\mathrm{COPD}, N(\%)$ & $27(11)$ & $21(10)$ & $6(15)$ & 0.404 & 0 \\
\hline Dementia, $N(\%)$ & $42(17)$ & $23(11)$ & $19(49)$ & $<0.001$ & 0 \\
\hline Number of chronic diseases (excluding dementia) & $2.0[1.0,3.0]$ & $2.0[1.0,3.0]$ & $3.0[2.0,4.0]$ & 0.008 & 0 \\
\hline Malnutrition & $74(35)$ & $58(32)$ & $16(50)$ & 0.069 & 12 \\
\hline Number of medications on admission & $6.0[2.0,8.0]$ & $5.0[2.0,8.0]$ & $6.0[4.0,8.0]$ & 0.134 & 0.8 \\
\hline Functional disability, $N(\%)^{* * *}$ & $98(42)$ & $70(35)$ & $28(74)$ & $<0.001$ & 2.1 \\
\hline Chest X-ray or CT results, $N(\%)$ & & & & 0.586 & 0 \\
\hline No opacity & $33(14)$ & $29(14)$ & $4(10)$ & & \\
\hline Single opacity & $134(56)$ & $109(54)$ & $25(64)$ & & \\
\hline Multiple or bilateral opacities & $74(31)$ & $64(32)$ & $10(26)$ & & \\
\hline CPAP on admission & $30(12)$ & $25(12)$ & $5(13)$ & 1 & 0 \\
\hline \multicolumn{6}{|l|}{ Laboratory indicators } \\
\hline White blood cell count $\left(\times 10^{9} / 1\right)$ & $6.2[4.7,9.2]$ & $6.2[4.8,9.2]$ & $6.1[4.6,10.2]$ & 0.945 & 0.4 \\
\hline c-reactive protein, $\mathrm{mg} / \mathrm{dl}$ & $4.9[1.6,8.9]$ & $4.9[1.4,8.7]$ & $4.5[2.1,10.9]$ & 0.343 & 0.4 \\
\hline Albumin gr/dl & $3.2[2.8,3.6]$ & $3.3[2.8,3.6]$ & $3.2[3.0,3.5]$ & 0.782 & 56 \\
\hline Days of delirium symptoms & $0.0[0.0,3.0]$ & $0.0[0.0,2.0]$ & $4.0[2.0,6.5]$ & $<0.001$ & 0 \\
\hline Length of stay (days) & $12.0[7.0,22.0]$ & $12.0[7.0,22.0]$ & $13.0[7.0,19.0]$ & 0.603 & 0 \\
\hline $\begin{array}{l}\text { Percentage of days of delirium symptoms/days of } \\
\text { hospital stay }\end{array}$ & $0.0[0.0,23.5]$ & $0.0[0.0,16.3]$ & $50.0[6.3,67.3]$ & $<0.001$ & 0 \\
\hline Mortality & $77(32)$ & $57(28)$ & $20(51)$ & 0.008 & 0 \\
\hline
\end{tabular}

CPAP: Continuous positive airway pressure; COPD: chronic obstructive pulmonary disease

*Median [IQR] where not otherwise specified

${ }^{* *}$ Cardiac diseases included congestive heart failure, coronary heart diseases and atrial fibrillation

*** Functional disability was defined according to the presence of dependency in self-bathing or self-dressing or with a Barthel Index score $\leq 90 / 100$ one month before the index hospitalization

delirium on admission was associated with almost a twofold increase in the risk of in-hospital mortality [11]. In the last years, researchers have further investigated how delirium is associated with worse outcomes not only when considered as a single episode but even more when the severity and the duration of delirium is measured [8-10]. Indeed several studies have emphasized the importance of delirium monitoring with validated tools not only at the time of admission to a single setting but also on a daily basis, since each day of delirium increases the risk of mortality by $10-17 \%$ [8-10].

Given the difficulties in spreading the practice of delirium monitoring in the daily routine, researchers have also investigated the possibility to detect delirium symptoms using a validated method in geriatric and medical wards [13]. This method has been applied also in postacute care and pulmonary wards $[16,17]$. Using this method, the prevalence of delirium symptoms in patients with severe pneumonia was
$31 \%$ and the presence of at least one delirium symptoms during the hospitalization was associated with a more than twofold increase in the odds of one-year mortality [17]. In our study, we found a significant association between the duration of delirium symptoms and in-hospital mortality in older patients with SARS-COV2 infection. These findings support previous investigations reporting the association between delirium duration and increased mortality [8-10].

The emergency related to the SARS-COV2 pandemic has determined a rapid subversion of the health care system organization, which, of course, did not spare the geriatric wards. Usually, a patient admitted to a geriatric ward is evaluated with a comprehensive geriatric assessment including delirium evaluation using validated tools such as the Confusion Assessment Method (CAM) [19] or the 4AT [14]. With the SARS-COV2 emergency, medical staffs were profoundly redesigned and specialists with a different 
Table 2 Patients' characteristics according to in-hospital death

\begin{tabular}{|c|c|c|c|c|}
\hline Variable* & $\begin{array}{l}\text { Alive } \\
N=164\end{array}$ & $\begin{array}{l}\text { Dead } \\
N=77\end{array}$ & $p$ value & Missing (\%) \\
\hline Age (years) & $74.4[62.1,83.0]$ & $83.3[76.9,89.0]$ & $<0.001$ & 0 \\
\hline Gender (male), $N(\%)$ & $91(55)$ & $52(68)$ & 0.092 & 0 \\
\hline Smoking (current or previous), $N(\%)$ & $36(29)$ & $25(47)$ & 0.026 & 27 \\
\hline Cardiac diseases, $N(\%)^{* *}$ & $47(29)$ & $46(60)$ & $<0.001$ & 0 \\
\hline Hypertension, $N(\%)$ & $89(54)$ & $54(70)$ & 0.024 & 0 \\
\hline Diabetes mellitus, $N(\%)$ & $33(20)$ & $24(31)$ & 0.074 & 0 \\
\hline $\mathrm{COPD}, N(\%)$ & $15(9)$ & $12(16)$ & 0.187 & 0 \\
\hline Dementia, $N(\%)$ & $21(13)$ & $21(27)$ & 0.01 & 0 \\
\hline Number of chronic diseases (excluding dementia) & $2.0[1.0,3.0]$ & $3.0[2.0,4.0]$ & $<0.001$ & 0 \\
\hline Malnutrition, $N(\%)$ & $39(27)$ & $35(53)$ & $<0.001$ & 12 \\
\hline Number of medications on admission & $4.0[1.0,7.0]$ & $7.0[5.0,9.5]$ & $<0.001$ & 0.8 \\
\hline Functional disability, $N(\%)^{* * *}$ & $53(33)$ & $45(61)$ & $<0.001$ & 2.1 \\
\hline Chest X-ray or CT results, $N(\%)$ & & & 0.005 & 0 \\
\hline No opacity & $30(18)$ & $3(4)$ & & \\
\hline Single opacity & $84(51)$ & $50(65)$ & & \\
\hline Multiple or bilateral opacities & $50(30)$ & $24(31)$ & & \\
\hline CPAP on admission, $N(\%)$ & $13(10)$ & $14(20)$ & 0.001 & \\
\hline \multicolumn{5}{|l|}{ Laboratory indicators (median [iqr]) } \\
\hline White blood cell count $\left(\times 10^{9} / 1\right)$ & $5.8[4.6,8.3]$ & $6.9[5.2,10.8]$ & 0.006 & 0.4 \\
\hline c-reactive protein, $\mathrm{mg} / \mathrm{dl}$ & $3.4[1.1,7.6]$ & $8.0[4.3,14.6]$ & $<0.001$ & 0.4 \\
\hline Albumin gr/dl & $3.2[2.8,3.6]$ & $3.3[3.0,3.5]$ & 0.845 & 56 \\
\hline Delirium on admission, $N(\%)$ & $19(12)$ & $20(26)$ & 0.007 & 0 \\
\hline Days of delirium symptoms & $0.0[0.0,2.0]$ & $2.0[0.0,4.0]$ & $<0.001$ & 0 \\
\hline $\begin{array}{l}\text { Percentage of days of delirium symptoms/days of hospital } \\
\text { stay }\end{array}$ & $0.0[0.0,7.7]$ & $22.2[0.0,60.0]$ & $<0.001$ & 0 \\
\hline Length of stay (days) & $14.0[8.0,25.2]$ & $7.0[5.0,13.0]$ & $<0.001$ & 0 \\
\hline
\end{tabular}

CPAP: Continuous positive airway pressure, COPD: chronic obstructive pulmonary disease

*Median [IQR] where not otherwise specified

${ }^{* *}$ Cardiac diseases included congestive heart failure, coronary heart diseases and atrial fibrillation

${ }^{* * *}$ Disability was defined according to the presence of dependency in self-bathing or self- dressing or with a Barthel Index score $\leq 90 / 100$ 1 month before the index hospitalization

background were added to the staff and called to comply with practices which are not typical of their expertise, such as delirium assessment. This change in the organization did not allow to always ensure the optimal approach to geriatric patients. Additionally, older patients are managed with protocols to prevent and treat delirium with an interdisciplinary and multidisciplinary approach [20]. Delirium assessment and prevention have been deeply affected, with obvious consequences in delirium management.

The study findings of an association between delirium symptoms and mortality should prompt clinicians and health care policy makers to consider how to approach the care of geriatric patients during an emergency, keeping the cardinal geriatric evaluations as to allow an adequate care. Within these evaluations, delirium monitoring should not be forgotten. One should be aware that tools such as the CAM or the 4AT might be difficult to be used during emergency situation and other screening tools for delirium can be used. For instance, the modified Richmond Agitation and Sedation Scale (m-RASS) [21] has been shown to have high specificity in delirium detection even in busy settings (i.e., emergency departments) and in patients with dementia [22]. The abnormal arousal level, as measured with the m-RASS, has also been shown to be associated with increased mortality at 1-month and 6-month follow-up [23]. The m-RASS has the advantage to be easy to use at the bedside and does not required a specific training allowing a daily monitoring of the possible presence of delirium.

Our study presents strengths along with limitations. This is the first multicenter study to report the association between delirium symptoms and in-hospital mortality in a relatively large cohort of elderly patients with SARS-COV2. In the current study, we used a previously validated methods for the detection of delirium symptoms via the review of 
Fig. 1 Violin plot and box-plot on the distribution of the percentage of days with delirium symptoms on hospital stay by in-hospital discharge status (dead, alive). Colored areas reports the kernel density in alive subjects (gray) and dead (orange). Box represents first and third quartile and the inner line indicates the median; the whiskers are located at the maximum and minimum observation (outside observations indicated with dots are those out of the $1.5 \times$ interquartile range). The figures shows that the majority of alive patients did not show any delirium symptoms during hospital stay, while patients who died had higher percentage of days with delirium symptoms

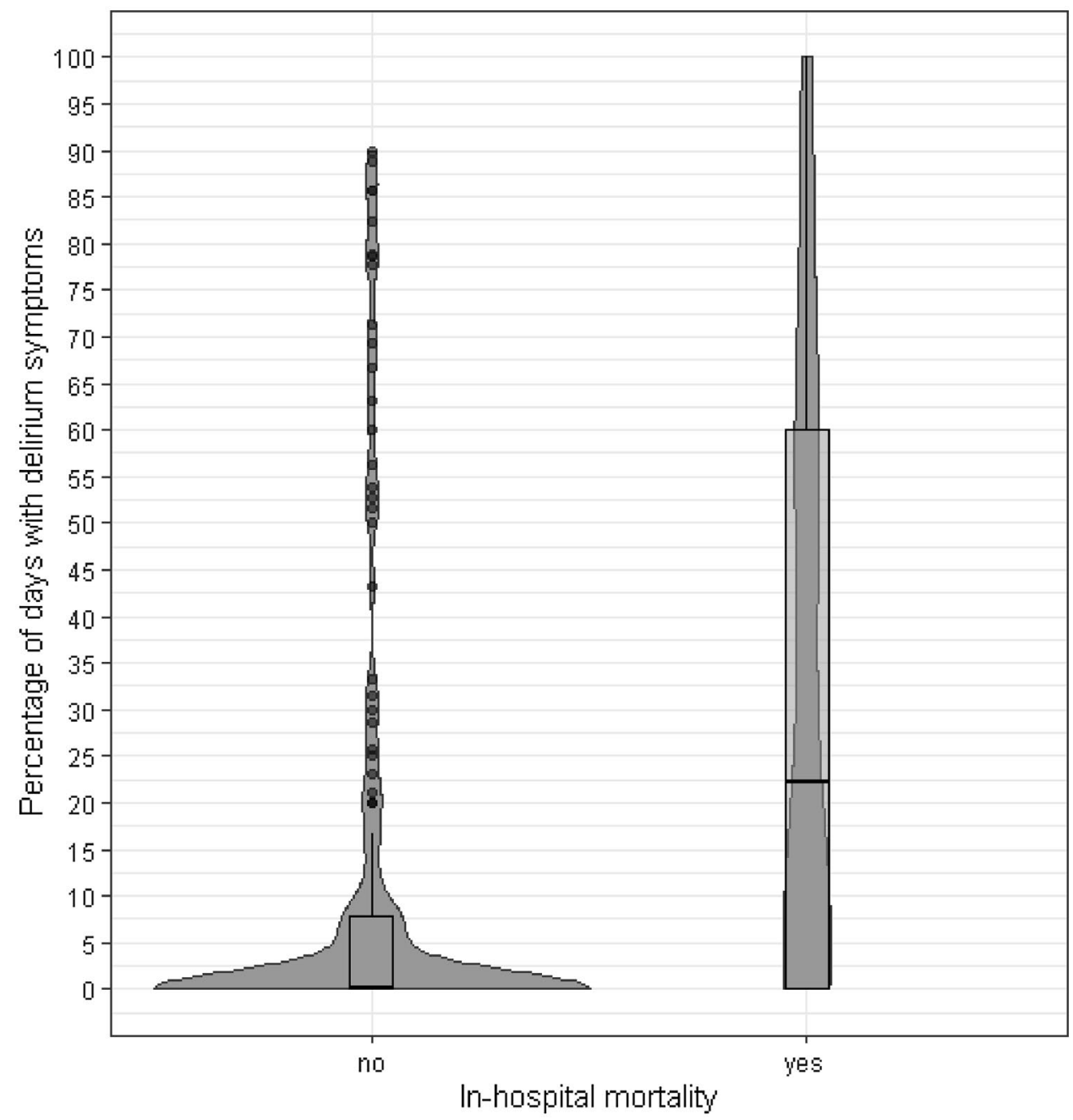

Table 3 Time-dependent multivariable Cox regression model stratified for center on the association between days of delirium symptoms and in-hospital mortality (238 patients, 68 events)

\begin{tabular}{lll}
\hline & HR $(95 \%$ CI $)$ & $p$ \\
\hline Days of delirium symptoms & $1.10(1.01 ; 1.2)$ & 0.0346 \\
Gender (male) & $1.63(0.89 ; 3.00)$ & 0.1133 \\
Age & $1.03(1.00 ; 1.06)$ & 0.0524 \\
Comorbidity & $1.24(1.03 ; 1.50)$ & 0.0258 \\
Cardiac disease* & $1.30(0.71 ; 2.38)$ & 0.3984 \\
COPD & $0.80(0.39 ; 1.65)$ & 0.5443 \\
CPAP on admission & $2.11(1.11 ; 4.02)$ & 0.0229 \\
Number of medications on admission & $1.13(1.04 ; 1.23)$ & 0.0034 \\
Bilateral lung opacity on admission & $3.05(0.87 ; 10.68)$ & 0.081 \\
Single lung opacity on admission & $3.07(0.86 ; 11.00)$ & 0.085 \\
CRP on admission & $1.08(1.04 ; 1.12)$ & 0.0001 \\
\hline
\end{tabular}

COPD: Chronic obstructive pulmonary disease; CPAP: Continuous positive airway pressure; CRP: C-reactive protein; HR: hazard ratio; CI: confidence interval

${ }^{*}$ Cardiac diseases included congestive heart failure, coronary heart diseases and atrial fibrillation medical records. The limitations include the retrospective design of the study and a non-formal assessment of delirium on daily basis with validated methods such as the CAM or the 4AT. However, this approach in an emergency situation allowed us to obtain results that are consistent with previous studies.

\section{Conclusions}

The duration of delirium symptoms in older patients with SARS-COV2 infection was found to be associated with a significant increase in in-hospital mortality. The study supports the necessity to establish protocols for the monitoring and management of delirium during emergency conditions to allow an appropriate care for older patients.

Supplementary Information The online version contains supplementary material available at https://doi.org/10.1007/s40520-021-01899-8.

Acknowledgements None. 
Authors' contributions Design (all authors); methods (all authors), subject recruitment (Morandi, Isaia, Grossi, Faraci, Gentile, Bo, Deiana, Ghezzi, Miksza, MD, Blangiardo, Bellelli), data collections (Morandi, Isaia, Grossi, Faraci, Gentile, Bo, Deiana, Ghezzi, Miksza, MD, Blangiardo, Bellelli), analysis and preparation of paper (all authors), final approval (all authors).

\section{Funding None.}

\section{Declarations}

Conflict of interest Dr. Morandi and Bellelli received Honoraria from Nestlè. The other authors do not report conflict of interests.

Ethical approval The study protocol was approved by the Brianza Institutional Review Board (protocol n. 2572).

Informed consent Verbal consent was obtained from all individual participants included in the study.

Statement of human and animal rights The study was conducted in accordance with the ethical standards of the institutional research committee and with the 1964 Helsinki Declaration and its later amendments or comparable ethical standards.

\section{References}

1. American Psychiatric Association. DSM-5 Diagnostic Classification. In: Diagnostic and Statistical Manual of Mental Disorders. 2013. https://doi.org/10.1176/appi.books.9780890425596.x00di agnosticclassification

2. Inouye SK, Westendorp RGJ, Saczynski JS (2014) Delirium in elderly people. Lancet. https://doi.org/10.1016/S0140-6736(13) 60688-1

3. Hawkins M, Sockalingam S, Bonato $S$ et al (2021) A rapid review of the pathoetiology, presentation, and management of delirium in adults with COVID-19. J Psychosom Res. https://doi.org/10. 1016/j.jpsychores.2020.110350

4. Bellelli G, Morandi A, Di Santo SG et al (2016) "Delirium Day": a nationwide point prevalence study of delirium in older hospitalized patients using an easy standardized diagnostic tool. BMC Med. https://doi.org/10.1186/s12916-016-0649-8

5. Morandi A, Di Santo SG, Zambon A et al (2019) Delirium, dementia, and in-hospital mortality: the results from the Italian Delirium Day 2016, a national multicenter study. J Gerontol Ser A Biol Sci Med Sci. https://doi.org/10.1093/gerona/gly154

6. Morandi A, Davis D, Fick DM et al (2014) Delirium superimposed on dementia strongly predicts worse outcomes in older rehabilitation inpatients. J Am Med Dir Assoc. https://doi.org/ 10.1016/j.jamda.2013.12.084

7. Morandi A, Lucchi E, Turco R et al (2015) Delirium superimposed on dementia: a quantitative and qualitative evaluation of patient experience. J Psychosom Res. https://doi.org/10.1016/j. jpsychores.2015.07.010

8. Pisani MA, Kong SYJ, Kasl SV, Murphy TE, Araujo KLB, Van Ness PH (2009) Days of delirium are associated with 1-year mortality in an older intensive care unit population. Am J Respir Crit Care Med. https://doi.org/10.1164/rccm.200904-0537OC
9. González M, Martínez G, Calderón J et al (2009) Impact of delirium on short-term mortality in elderly inpatients: a prospective cohort study. Psychosomatics. https://doi.org/10.1176/appi.psy. 50.3.234

10. Bellelli G, Mazzola P, Morandi A et al (2014) Duration of postoperative delirium is an independent predictor of 6-month mortality in older adults after hip fracture. J Am Geriatr Soc. https://doi.org/ 10.1111 /jgs. 12885

11. Rebora P, Rozzini R, Angelo B et al (2020) Delirium in patients with SARS-CoV -2 infection: a multicenter study. J Am Geriatr Soc. https://doi.org/10.1111/jgs.16969

12. Mcloughlin BC, Miles A, Webb TE et al (2020) Functional and cognitive outcomes after COVID-19 delirium. Eur Geriatr Med. https://doi.org/10.1007/s41999-020-00353-8

13. Inouye SK, Leo-Summers L, Zhang Y, Bogardus ST, Leslie DL, Agostini JV (2005) A chart-based method for identification of delirium: validation compared with interviewer ratings using the confusion assessment method. J Am Geriatr Soc. https://doi.org/ 10.1111/j.1532-5415.2005.53120.x

14. Bellelli G, Morandi A, Davis DHJ et al (2014) Validation of the 4AT, a new instrument for rapid delirium screening: a study in 234 hospitalised older people. Age Ageing 43:496-502. https:// doi.org/10.1093/ageing/afu021

15. Tieges Z, MacLullich A, Anand A et al (2020) Diagnostic accuracy of the 4AT for delirium detection: systematic review and meta-analysis. Age Ageing. https://doi.org/10.1101/2020.06.11. 20128280

16. Morandi A, Solberg LM, Habermann R et al (2009) Documentation and management of words associated with delirium among elderly patients in postacute care: a pilot investigation. J Am Med Dir Assoc 10:330-334

17. Aliberti S, Bellelli G, Belotti M et al (2015) Delirium symptoms during hospitalization predict long-term mortality in patients with severe pneumonia. Aging Clin Exp Res. https://doi.org/10.1007/ s40520-014-0297-9

18. Marengoni A, Zucchelli A, Grande G, Fratiglioni L, Rizzuto D (2020) The impact of delirium on outcomes for older adults hospitalised with COVID-19. Age Ageing. https://doi.org/10.1093/ ageing/afaa189

19. Inouye SK (1990) Clarifying confusion: the confusion assessment method. Ann Intern Med. https://doi.org/10.7326/ 0003-4819-113-12-941

20. SIGN Evidence-based clinical guidelines. Sign 157 Risk reduction and management of delirium: a national clinical guideline. Healthc Improv Scotl. 2019.

21. Chester JG, Beth Harrington M, Rudolph JL (2012) Serial administration of a modified richmond agitation and sedation scale for delirium screening. J Hosp Med. https://doi.org/10.1002/jhm.1003

22. Morandi A, Han JH, Meagher D et al (2016) Detecting delirium superimposed on dementia: evaluation of the diagnostic performance of the Richmond Agitation and Sedation Scale. J Am Med Dir Assoc. https://doi.org/10.1016/j.jamda.2016.05.010

23. Bellelli G, Mazzone A, Morandi A et al (2016) The effect of an impaired arousal on short- and long-term mortality of elderly patients admitted to an acute geriatric unit. J Am Med Dir Assoc. https://doi.org/10.1016/j.jamda.2015.10.002

Publisher's Note Springer Nature remains neutral with regard to jurisdictional claims in published maps and institutional affiliations. 